\title{
USO DA IOT, BIG DATA E INTELIGÊNCIA ARTIFICIAL NAS CAPACIDADES DINÂMICAS: Um estudo comparativo entre cidades do Brasil e de Portugal
}

\author{
USE OF IOT, BIG DATA AND ARTIFICIAL INTELLIGENCE IN \\ DYNAMIC CAPABILITIES:
}

A comparative study between the cities of Brazil and Portugal

Claudio Marcio Campos de Mendonça ${ }^{1}$ António Manuel Valente de Andrade ${ }^{2}$

\begin{abstract}
RESUMO
0 artigo teve como objetivo identificar as relações entre loT, Big Data e Inteligência Artificial com os microfundamentos das capacidades dinâmicas, em organizações localizadas na Cidade do Porto-Portugal e Natal-Brasil, e comparar os resultados obtidos nas duas localidades. A pesquisa caracteriza-se como exploratório e descritivo com abordagem quantitativa. Foram selecionados 190 questionários válidos, 53 questionários de Natal e 137 de Porto. A pesquisa realizada foi relevante para comparar o desenvolvimento empresarial tecnológico em Portugal e no Brasil, demonstrando como está ocorrendo 0 processo de transformação digital nas diferentes localidades, e como essa transformação contribui para as capacidades dinâmicas. Foi observado que a tecnologia Big Data obteve maior percentual de desempenho atual em comparação as tecnologias IoT e IA. Ademais, a tecnologia IA, foi a ferramenta tecnológica que obteve as menores médias nas três capacidades dinâmicas avaliadas (sensing, seizing e managing threats), sendo pouco utilizado, principalmente em Natal. Analisando de forma macro, as empresas localizadas em Porto se sobressaem em comparação às localizadas em Natal, em todas as dimensões e capacidades. Contudo, quando avaliado a importância das tecnologias, atual e futura, foi detectado um potencial de desenvolvimento futuro no desempenho em todas as categorias analisadas, tanto em Porto como em Natal. Palavras-chave: Transformação Digital. Capacidades Dinâmicas. Internet das coisas. Big Data. Inteligência artificial.
\end{abstract}

\section{ABSTRACT}

The article had the goal to identify the relationships between IoT, Big Data and Artificial Intelligence with the dynamic capabilities in micro-foundations, with organizations located in Porto-Portugal and Natal-Brazil, and to compare the results obtained in those two locations. The research is characterized as exploratory and descriptive with a quantitative approach. Were selected 190 valid questionnaires, 53 questionnaires from Natal and 137 from Porto. This research was relevant to compare technological business development in Portugal and Brazil, demonstrating how the process of digital transformation is taking place in different locations, and how this transformation contributes to dynamic capabilities. It was observed that Big Data technology obtained the highest percentage of current performance compared to IoT and IA technologies. Besides that, Al technology was the technological tool that obtained the lowest averages in the three evaluated dynamic capacities (sensing, seizing and managing threats), being underused, especially in Natal. In General, those companies located in Porto stand out compared to those located in Natal, in all sizes and capacities. However, when assessing the importance of current and future technologies, the potential for future development in performance was detected in all categories analyzed, particularly in Porto and Natal.

Keywords: Digital Transformation. Dynamic Capabilities. Internet of Things. Big Data. Artificial Intelligence

1 Doutor em Administração pela Universidade Federal do Rio Grande do Norte, Brasil. Professor do Magistério Superior da Universidade Federal do Amapá, Brasil. E-mail: cmarcio@gmail.com.

2 Doutor em Tecnologias e Sistemas de Informação pela Universidade do Minho, Portugal. Professor Auxiliar na Universidade Católica Portuguesa, Portugal. E-mail: aandrade@porto.ucp.pt. 


\section{INTRODUÇÃ̃}

As evoluções das tecnologias digitais estão modificando o processo industrial, e não somente a indústria é afetada por essa evolução, por esse motivo, é possível afirmar que hoje estamos no processo de uma revolução, a quarta revolução industrial ou indústria 4.0, que irá alterar a economia mundial, além da forma como vivemos e nos relacionamos, através de inovações tecnológicas (COELHO, 2016; SCHWAB, 2016). Caraffini, Souza e Behr (2017) afirmam que as organizações estão aderindo tecnologias como computação em nuvem, inteligência artificial e outras tecnologias, com propósito de aumentar a satisfação do cliente e sua eficiência nos serviços. As empresas estão se adaptando às novas configurações de mercado para obter notoriedade na economia, e para isso é necessário rapidez e agilidade (SCHWAB, 2016).

As tecnologias da transformação digital, Big Data, Computação em Nuvem, Internet das Coisas e Inteligência Artificial são, atualmente, as tecnologias base que sustentam a inovação tecnológica. Portanto, para que as empresas acompanhem o processo da indústria 4.0 estão fazendo uso dessas tecnologias para desenvolver e/ou aprimorar as capacidades dinâmicas empresariais. Guerra, Tondolo e Camargo (2016) definem as capacidades dinâmicas como a capacidade de identificar inovações no mercado através de microfundamentos e implementá-las nos processos base da organização, reconfigurando processos operacionais. As tecnologias da transformação digital combinadas com as capacidades dinâmicas são capazes de aumentarem a produtividade e garantir vantagem competitiva.

Assim sendo, o presente estudo faz o seguinte questionamento: quais são as relações entre os elementos da transformação digital (IoT, Big Data e IA) com os microfundamentos das capacidades dinâmicas em empresas Portuguesas e Brasileiras? Diante da problemática, a pesquisa tem como objetivo identificar as relações entre IoT, Big Data e Inteligência Artificial com os microfundamentos das capacidades dinâmicas, em organizações localizadas na Cidade do Porto-Portugal e Natal-Brasil, e comparar os resultados obtidos nas duas localidades. 0 artigo é composto pelo referencial teórico que aborda as questões relacionadas a transformação digital e suas tecnologias (IoT, Big Data e IA) e as capacidades dinâmicas. A metodologia definida para se alcançar o objetivo proposto. Além da análise e interpretação dos resultados e considerações finais. 


\section{REFERENCIAL TEÓRICO}

\subsection{Quarta revolução industrial e transformação digital}

A temática da quarta revolução industrial vem ganhando espaço nas publicações científicas, desde que a terminologia "Industry 4.0" foi utilizada de forma mais marcante em 2011, na Feira de Hannover (Alemanha), através de uma associação de representantes de negócios, política e academia, que tinha como objetivo fortalecer a competitividade da indústria Alemã (HERMANN; PENTEK; OTTO, 2015; SCHWAB, 2016). Sendo esse o país que mais produz trabalhos científicos nessa área de conhecimento, 45,29\% das publicações na plataforma Web of Science sobre a indústria 4.0, são de origem alemã (YANAl et al., 2017).

0 modelo produtivo anterior está em processo de ruptura, e que a quarta revolução industrial, é 0 elemento que vem gerando esse processo de forma rápida. A quarta revolução industrial, tratase na sua essência da fusão de tecnologias como: sistemas e máquinas inteligentes e conectadas; nanotecnologia; energias renováveis; e computação quântica, e a interação entre os domínios físicos, formados por veículos autônomos, impressão em 3D, robótica avançada e novos materiais; digitais, com elementos como a computação em nuvem, internet das coisas (loT) e a inteligência artificial; e por último o biológico, composto pela biologia sintética e engenharia genética (SCHWAB, 2016).

Da categoria digital, alguns elementos como a computação em nuvem, IoT, Inteligência Artificial e Big Data, fazem parte da denominada Transformação Digital, que é uma perspectiva de uso da tecnologia da informação e da comunicação (TIC), onde ela vem a atuar como elemento preponderante na transformação e reconfiguração de elementos organizacionais, tais como: estratégia, processos, cultura e estruturas (HESS et al., 2016). As tecnologias da transformação digital estão interconectadas, visto que possuem uma inter-relação de dependência para obterem maior aproveitamento (SCHWAB, 2016).

"A crescente competitividade e velocidades das mudanças têm tornado a inovação tecnológica fundamental para a vantagem competitiva" (R0TTA, 2011, P 140). A transformação digital muda as regras de competitividade, o diferencial agora se encontra na capacidade de renovação constante e ágil, está fortemente focalizada na eficiência, produtividade, segurança e lucro dos investimentos (COELHO, 2016). Assim sendo, para obter notoriedade é necessário um aprendizado contínuo, flexibilidade para se adaptar, rapidez para corresponder às expectativas, além de se auto impor desafios (SCHWAB, 2016). 
Um estudo realizado por Caraffini, Souza e Behr (2017) demonstrou que a transformação digital afetou grandemente e de forma positiva as organizações financeiras que aderiram tecnologias como computação em nuvem e inteligência artificial, maximizando sua eficiência e satisfação do público alvo, diversas organizações estão aderindo às essas e outras tecnologias com o mesmo propósito, utilizando inclusive iniciativas para aumentar a inovação com o apoio de parceiros externos.

\subsection{Big Data e computação em nuvem}

0 Big Data está emergindo como um tema relevante entre estudiosos e profissionais, e é definido como uma abordagem holística para gerenciar, processar e analisar os dados em cinco dimensões. 0 Big Data permite a melhora da eficiência e eficácia das organizações, e permite o processo de tomada de decisão com base em evidências e não intuição (MCAFEE; BRYNJOLFSSON, 2012). É uma solução originada devido à imensidão de dados que são gerados diariamente, dados esses que aumentam exponencialmente de acordo com a evolução tecnológica, é a forma mais eficiente até então para obter informações importantes ao mercado competitivo (HENRIQUES et al., 2013).

As dimensões do Big Data são denominadas de "5Vs", são elas: Volume - satisfação, acessibilidade aos dados; Variedade - diversidade de fontes e tipos de dados; Velocidade - tempo de acesso à informação e a tomada de decisão; Veracidade - confiança na exatidão apresentada pelos dados; e Valor - o que as informações melhoram nos resultados, valor financeiro utilizado para conseguir obter dados com um bom nível de qualidade (BRAGANZA et al., 2017; JUNIOR et al., 2015; MAÇADA; CANARY, 2014; MCAFEE; BRYNJOLFSSON, 2012; SIVARAJAH et al., 2017; VENKATRAM; GEETHA, 2017; WAMBA et al., 2017).

Tessarolo e Magalhães (2014) e Caldas e Silva (2016) classificam os dados em estruturados e não estruturados, em que os estruturados representam as informações organizadas e classificadas, já os não estruturados representam os desordenados, que não possuem grande utilidade se não os ordenar e interpretá-los, devido ao grande volume e rapidez com que são gerados, somente $10 \%$ desses dados são estruturados e 90\% são não estruturados. Novo e Neves (2013) apontam a necessidade de rapidez e agilidade como um fator decisivo na análise e implementação dos dados obtidos, para aperfeiçoar setores e processos organizacionais.

De acordo Novo e Azevedo (2014) o Big Data está atuando principalmente nas mídias sociais onde as informações geradas são utilizadas para direcionar conteúdo específicos de acordo 
com os interesses dos usuários. No entanto, os usuários das plataformas digitais têm manifestado dúvidas e preocupações quanto à segurança e privacidade dos dados (BORBA; SANTOS; JUNIOR, 2013; BRETERNITZ; SILVA; LOPES, 2013; PIMENTA, 2013; NOVO; AZAVED0, 2014; SCHROEDER, 2018). Sendo assim, é necessário ressaltar-se a importância de uma boa conduta ética por parte da gestão organizacional para com seu público (BRETERNITZ; SILVA; LOPES, 2013; NOVO; AZEVEDO, 2014).

Em suma, o Big Data vem emergindo com uma poderosa ferramenta tecnológica nos dias atuais, pois dentre outras coisas, vem permitindo a evolução do processo decisório. Ele permite analisar 0 universo de dados em vez de alguns dados de amostra, portanto, não há resultados tendenciosos. 0 exemplo ocorre com as redes sociais onde se permite análises de forma muito econômica e um feedback das opiniões dos clientes de forma mais rápida. É possível construir um produto com base nas preferências dos clientes com a ajuda de Big Data (VENKATRAM; GEETHA, 2017). 0 atual volume e rapidez com que o Big Data é gerado diariamente requer uma boa ferramenta de armazenamento, portanto a computação em nuvem tem sido a ferramenta mais indicada devido ao seu poder de escalabilidade e flexibilidade sem haver necessidade de ocupar espaço físico e ao baixo custo visto devido à forma de cobrança (SILVA et al., 2013).

A CN tornou-se um componente essencial para implementação de estratégias na qual a área de TI é demandada (SANTOS; AMELOTTI; VILLAR, 2012). De acordo com Oliveira et al. (2013) e Vieira e Meirelles (2015) a CN está cada vez mais presente no dia a dia da sociedade representando uma revolução na área da computação. Sendo uma ferramenta amplamente utilizada no meio organizacional, no âmbito científico assim como pela população doméstica também (SOUSA; MOREIRA; MACHADO, 2009; BORGES et al., 2011; PEDROSA; NOGUEIRA, 2011).

A computação em nuvem, na sua conceituação, tem alguns aspectos em comum abordados por vários autores, como: a redução de custo, a padronização dos serviços de TI, a maior facilidade de mensuração, o pagamento somente do que realmente se utilizou, acesso a inovação, disponibilidade imediata de recursos e a escalabilidade. Além de fatores como: confiabilidade, escalabilidade, economia, privacidade e segurança (BADGER; PATT-CORNER; VOAS, 2011; LUI et al., 2011; MARSTON et al., 2011; MELL; GRANCE, 2010; VERAS, 2012). Ainda que exista diversas vantagens no uso da CN ainda se percebe relutância de alguns usuários com relação aos desafios que a tecnologia ainda encontra com relação à segurança e privacidade (VIEIRA; MEIRELLES, 2015; SOUSA; MOREIRA; MACHADO, 2009; MACED0, 2015; OLIVEIRA et al., 2013). 
Dias (2013) relata que apesar da insegurança existente é inegável os benefícios em dispor de um banco de dados armazenados em nuvem. É superior em benefícios em diversos sentidos com relação ao modelo tradicional, incentivando assim o seu desenvolvimento e aperfeiçoamento (BORGES et al., 2011). Para Macedo (2015), Pedrosa e Nogueira (2011) a confiabilidade do usuário, não diretamente para com a tecnologia, mas sim com provedor da mesma, é um grande influenciador na adesão do uso e na popularização da CN, concluindo que são fatores intrínsecos que determinam a sua utilização ou não, como a credibilidade do fornecedor e a praticidade na utilização.

\subsection{Internet das coisas (IOT) e inteligência artificial (IA)}

Diante da tecnologia da Computação em Nuvem e da sua junção com tecnologias como sistemas embarcados, microeletrônica, comunicação e sensores, surge o conceito de Internet das Coisas, oriunda do inglês Internet of Things (IoT). Termo utilizado primeiramente por Ashton (2009) ao trabalhar com conceito de RFID (Radio Frequency Identification).

A loT é uma inovação tecnológica que trouxe inteligência aos objetos, ou como afirmam Zhu, Leung, Shu, \& Ngai (2015), é um facilitador inteligente do mundo, que tem na sua estrutura, artefatos como a Internet e objetos inteligentes (GALEGALE et al., 2016). As tecnologias de identificação de rádio frequência (RFID) e de sensores, evoluirão ainda mais para enfrentar este novo desafio no qual os sistemas de informação e comunicação estão invisivelmente integrados no ambiente ao nosso redor (GUBBI et al., 2013).

Já Van Kranenburg e Anzelmo (2008) percebem que a loT surge como uma inovação tecnológica que vem trazendo grandes mudanças para a gestão da cadeia de suprimentos. Onde 0 objetivo é conectar vários objetos com endereços exclusivos, para que possam interagir um com 0 outro, e também com o mundo (DINH; KIM; LEE, 2017; GUBBI et al., 2013; ZHU et al., 2015). Para Macadar e Garcia (2017), a IoT sendo uma inovação tecnológica capaz de geração, processamento e transmissão de dados sobre as coisas e seu ambiente, torna a área da gestão da informação ainda mais complexa. Além de influenciar em áreas como aprendizagem, comportamento, tomada de decisão, análise de riscos, gestão de recursos e conflitos.

Nos últimos anos, há visivelmente uma evolução dos artefatos tecnológicos que circundam a sociedade nas suas mais variadas perspectivas, para Makridakis (2017) um desses artefatos é a IA, e ele afirma que o impacto das revoluções industrial e digital ou da informação, afetou substancialmente 
a sociedade, mas há uma nova revolução sendo formada pela Inteligência Artificial (IA) e que gerará forte impacto nas empresas e empregos.

A IA hoje está presente no dia-a-dia das pessoas e empresas, um exemplo disso são recursos de reconhecimento de voz, de face e sugestões de escrita disponíveis nos smartphone de hoje, as pessoas poderão comprar bens e obter serviços de qualquer lugar do mundo usando a Internet e explorar os benefícios ilimitados da IA, pois computadores e os robôs provavelmente se aproximarão da inteligência humana nos próximos 20 anos (MAKRIDAKIS, 2017).

A IA é um vasto campo de pesquisa, tendo várias abordagens diferentes, dentre elas: a teoria fuzzy; árvores de decisão; e redes neurais. Elas vêm sendo utilizadas em vários tipos de aplicações em áreas diferentes (KLASHANOV, 2016; WOLFERT et al., 2017). E essas abordagens basicamente têm como foco ensinar os computadores a pensar por si mesmos e a improvisar soluções para problemas comuns, dessa forma a IA não somente aplica decisões pré-programadas, mas sim, exibe algumas capacidades de aprendizado (MAKRIDAKIS, 2017).

De acordo com Schwab (2016), a inteligência artificial e a robótica, gerarão uma reviravolta nas organizações com relação às funções administrativas nas quais ela passará a ocupar. Essa introdução da IA cada vez mais no mercado e nas organizações, tem como aspectos positivos a redução de custos, ganhos de eficiência e a facilidade de pequenas empresas que exploram fortemente 0 aspecto da inovação, mas pontos negativos também são observados, tais como: perda de postos de trabalho, prestação de contas e responsabilidade, mudanças legais, de relatos financeiros e riscos.

\subsection{Capacidades dinâmicas (dynamic capabilities)}

As Capacidades Dinâmicas (CD) ou Dynamic Capabilities, vêm sendo vistas como um acontecimento nas organizações e despertando interesse no ambiente acadêmico, bem como no corporativo nos seus mais diversos segmentos, que vão desde a área estratégica, passando pela gestão de recursos humanos, marketing, inovação, empreendedorismo e gestão da informação e do conhecimento.

De acordo com Helfat e Peteraf (2009) o termo Capacidade Dinâmica é relativamente recente, portanto ainda não é considerado como uma teoria. Estudos acerca do assunto ainda estão sendo desenvolvidos, fazendo com que esteja sujeito à alterações, ampliações e ramificações (GUERRA; TONDOLO; CAMARGO, 2016; TONDOLO; BITENCOURT, 2014). Tondolo e Bitencourt (2014) reafirmam 
a importância da realização de estudos futuros para identificar novas categorias e formas de análise. Barreto (2010) afirma ser necessário uma consolidação do conceito de capacidades dinâmicas antes que outras etapas de pesquisa sejam tomadas no campo para garantir que consideradas suposições, variáveis e relacionamentos adequados.

As capacidades dinâmicas são constituídas por três elementos, que são os comportamentos e habilidades, as rotinas e processos, os mecanismos de aprendizagem e governança do conhecimento, baseando-se em duas visões, de que a CD é gerada a partir de uma aglomeração de habilidades, hábitos comportamentais e capacidades organizacionais e afirma que as CDs são processos e rotinas que a organização utiliza para se adaptar ao macro ambiente e obter vantagem competitiva (MEIRELLES; CAMARG0, 2014). Galdo (2016) refere-se às CDs diferentemente dos outros autores considerandoas como um recurso intangível de uma organização, sendo habilidades, processos estratégicos, competências que possibilitam o desenvolvimento, mudança, adaptação, configuração e reconfiguração de suas atividades bases em resposta às demandas do ambiente externo, visando a geração de valor e maior competitividade.

Para os primeiros autores a abordar essa temática, Teece et al. (1997), a capacidade dinâmica consiste na habilidade de uma firma, de forma intencional, integrar, construir e reconfigurar suas competências, sejam elas, internas e externas, para se adequar aos ambientes dinâmicos. A questão central das capacidades dinâmicas está no processo através do qual desenvolve e renova as competências existentes da organização, com base em designações/decisões passadas internamente e do ambiente dinâmico.

Ascapacidades dinâmicas possuem como principal benefício a vantagem competitiva (BALESTR0 et al., 2004; ROTTA, 2011; MEIRELLES; CAMARGO, 2014; TONDOLO; BITENCOURT, 2014; GALDO, 2016; PEREIRA, 2018). Rotta (2011) acrescenta que as CDs possuem forte poder mediador entre as mudanças do mercado com as estratégias competitivas, as capacidades de absorção, adaptação, inovação e integração são as principais capacidades que deverão ser consideradas no momento de tomada de decisão, Herrmann (2013) ressalta a influência de direcionar uma organização em seu ajustamento estratégico contínuo e para Balestro et al. (2004) as CDs são de relevante importância no processo de adoção e desenvolvimento da inovação colaborativa, inovação essa tornou-se uma das principais formas para aumentar a vantagem competitiva.

Já Eisenhardt \& Martin (2000), discorrem a relevância dos processos para as capacidades dinâmicas, para eles, são os processos da empresa que utilizam recursos, especialmente os 
processos, são usados para integrar, reconfigurar, obter e liberar recursos, para combinar e até mesmo criar mudanças de mercado. Uma característica relevante é que a CD aborda, é a questão da rápida capacidade adaptativa das organizações perante um mercado competitivo e a busca pela vantagem competitiva nesse cenário variável com mudanças, sobretudo, tecnológicas (ARNDT; NORBERT, 2015; BARRETO, 2010; MEIRELLES; CAMARGO, 2014; D. J. TEECE, 2007; D. J. TEECE et al., 1997).

De acordo com Pereira (2018) as condições internas da organização combinadas com 0 monitoramento do ambiente externo geram indicativos de conhecimentos e esses indicativos possibilitam novos meios de reconfiguração; por meio da aprendizagem organizacional; para melhor ajuste da organização. Teece (2007), afirma a necessidade da existência de algumas capacidades que estão relacionadas com processos dos microfundamentos das capacidades dinâmicas, e que são desagregadas em três categorias organizacionais com os seguintes objetivos: atuar analisando o ambiente externo na qual a firma está inserida; aproveitar as chances/oportunidades detectadas; e gerenciar ameaças e transformações decorrentes das mudanças organizacionais. No quadro 1, são apresentadas as descrições dos processos que fazem parte dos microfundamentos das capacidades dinâmicas de acordo com Teece (2007).

Quadro 1 - Microfundamentos das capacidades dinâmicas e seus principais processos

\begin{tabular}{|c|l|}
\hline \multicolumn{1}{|c|}{ Capacidades } & \multicolumn{1}{c|}{ Processos } \\
\hline $\begin{array}{c}\text { Capacidade de atuar analisando o ambiente } \\
\text { externo na qual está inserida (Sensing) }\end{array}$ & $\begin{array}{l}\text { Processos para direcionar trabalhos internos de P\&D; processos } \\
\text { de buscar fornecedores para complementar as inovações da } \\
\text { organização; processos para buscar desenvolvimentos científicos } \\
\text { e tecnológicos externos à firma; processos para reconhecer } \\
\text { segmentos de mercado alvo, mudando as necessidades dos clientes } \\
\text { e gerando inovações direcionadas para os clientes. }\end{array}$ \\
\hline $\begin{array}{l}\text { Capacidade de aproveitar as chances/ } \\
\text { oportunidades detectadas (Seizing) }\end{array}$ & $\begin{array}{l}\text { Processos voltados para soluções para o cliente; processos de } \\
\text { de protocolos de tomada de decisões; processos para construir } \\
\text { lealdade de comprometimento; processos de descentralização e } \\
\text { decomposição; }\end{array}$ \\
\hline $\begin{array}{l}\text { Capacidade de gerenciar ameaças e } \\
\text { transformações decorrentes das mudanças } \\
\text { organizacionais - reconfiguração (Managing } \\
\text { threats/transforming) }\end{array}$ & $\begin{array}{l}\text { Processos de coespecialização; processos de } \\
\text { processos de gerenciamento do conhecimento. }\end{array}$ \\
\hline
\end{tabular}

Fonte: Adaptado de Teece (2007) 
Conforme afirma Teece (2007), embora o desempenho de longo prazo da empresa seja determinado, de alguma forma pelo modo como o ambiente empresarial (externo) recompensa seu patrimônio, o desenvolvimento e o exercício de capacidades dinâmicas (internas), estão no centro do sucesso empresarial ou na sua falha. A crescente demanda de inovação baseada na tecnologia da informação e a busca pela vantagem competitiva fizeram com que as CDs se tornassem necessárias para aumentar qualitativamente o desempenho organizacional (BALESTR0 et al., 2004).

\section{METODOLOGIA}

0 presente estudo se caracterizou como exploratório e descritivo, devido buscar identificar possíveis relações e diferenças entre um conjunto de variáveis, e sem necessariamente se preocupar com o quesito da causalidade. Quanto à natureza da pesquisa e abordagem ao problema, foi feita uma pesquisa quantitativa, utilizando o método de pesquisa survey descritiva. Quanto ao momento de coleta da pesquisa, foi utilizado o corte transversal (cross-sectional study) em um só momento cuja pretensão foi de descrever e analisar o estado das variáveis em um dado momento.

Amostragem utilizada foi a não-probabilística, e seus elementos foram selecionados de acordo com a conveniência do pesquisador. Para a aplicação dos questionários com questões fechadas e uma aberta, foi utilizado um instrumento eletrônico de aplicação de questionários. 0 link foi enviado por email, de forma Institucional, pela Universidade Católica do Porto, através da sua Newsletter, para os membros da Associação de Antigos Alunos da Faculdade de Economia e Gestão da Católica Porto Business School, o link foi enviado também para profissionais das áreas de TI e Administração do Rio Grande do Norte e foi encaminhado inclusive para grupos fechados no Facebook de profissionais das duas áreas. Foram respondidos ao todo 319 questionários, 72 questionários aplicados em Natal e 247 aplicados em Porto, que após análise e exclusão de questionários preenchidos de forma incompleta e retirados os questionários respondidos por pessoas que não eram gestores de negócio ou TI, restaram 190 questionários válidos, 53 questionários de Natal e 137 de Porto.

Um teste piloto do questionário foi enviado para 2 alunos de doutorado em administração com conhecimento na área de tecnologia e inovação. Também o instrumento foi submetido a avaliação de 2 doutores com experiência em instrumentos quantitativos. Após as sugestões, as considerações foram acatadas e implementadas no instrumento de pesquisa. 
Foi utilizada na pesquisa uma adaptação da matriz de importância $x$ desempenho/performance de Martilla e James (1977), e em parte do modelo acrescentada uma dimensão denominada de importância futura na percepção dos respondentes, pois os elementos da transformação digital, conforme afirmam Kane et al. (2015), ainda se encontram em fase de amadurecimento nas organizações e essa terceira dimensão trará a perspectiva de importância dessas tecnologias sobre as capacidades dinâmicas nos próximos 7 anos, ou seja, até 2025, ano que serão detectados, segundo Schwab (2016), alguns pontos de inflexão da quarta revolução industrial.

Para Malhotra (2001), a primeira providência empírica adotada foi a determinação da confiabilidade de consistência interna da escala do tipo-Likert, através do Alfa de Cronbach e que esse coeficiente varia de 0 a 1 e indica confiabilidade insatisfatória de consistência interna quando fica igual ou menor que 0,6. 0 alfa de Cronbach obtido foi relativamente alto para as questões que envolvem as capacidades dinâmicas, sendo 0,925 para as questões aplicadas em Natal e 0,92 para as plicadas em Porto com 9 questões das 3 tecnologias, totalizando 27 itens com escala do tipo-Likert. Quando separados por categorias, em Natal foi obtido o valor de 0,925 para questões relativas a loT, 0,945 para Big Data e 0,878 para Inteligência Artificial, em Porto foi obtido o valor de 0,909 para questões relativas a IoT, 0,919 para Big Data e 0,934 para Inteligência Artificial.

\section{ANÁLISE E INTERPRETAÇÃO DOS RESULTADOS}

\subsection{Perfil}

Os primeiros resultados demonstraram uma paridade entre o porte das organizações dos participantes da pesquisa. 0 percentual de empresa dominante corresponde a grandes empresas, sendo correspondente a quase metade dos entrevistados em ambas as cidades. 0 porte de empresa que menor teve participação na pesquisa foi de pequena empresa, correspondendo a 13,9\% em na cidade do Porto e 15,1\% na cidade de Natal, conforme demonstra o quadro 2. 
Quadro 2 - Porte da Empresa

\begin{tabular}{|l|c|c|c|c|}
\hline & Porte & & & \\
Cidade & Grande & Média & Pequena & Micro \\
\hline Porto & & & & \\
\hline Natal & $49,6 \%$ & $16,1 \%$ & $13,9 \%$ & $20,4 \%$ \\
\hline
\end{tabular}

Fonte: Dados da pesquisa (2019)

Dessas empresas, os resultados apontaram uma diferença significativa entre a origem das mesmas no comparativo das cidades. No quadro 3 , na cidade do Porto, o maior percentual corresponde a empresas multinacionais $(59,1 \%)$, enquanto na cidade de Natal, o maior percentual está concentrado em empresas nacionais $(67,9 \%)$.

Quadro 3 - Origem da empresa

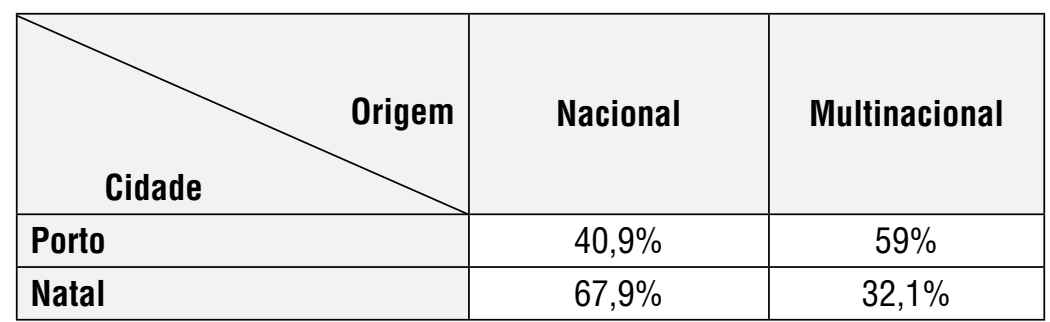

Fonte: Dados da pesquisa (2019)

A respeito do tipo de entrega que essas empresas fazem a seus clientes, há certa diferença entre o percentual dominante em cada cidade. A oferta de só "serviço" é predominante na cidade do Porto, enquanto na cidade de Natal essa predominância está localizada na oferta de "produto e serviço", conforme apresenta o quadro 4.

Quadro 4 - Tipo de entrega

\begin{tabular}{|l|c|c|c|}
\hline \multicolumn{1}{|c|}{ Cidade } & Serviço & $\begin{array}{c}\text { Produto e } \\
\text { Serviço }\end{array}$ & Produto \\
\hline Porto & & & \\
\hline Natal & $35,3 \%$ & $31,4 \%$ & $23,4 \%$ \\
\hline
\end{tabular}

Fonte: Dados da pesquisa (2019) 
No quadro 5, os pesquisadores optaram por selecionar somente os questionários respondidos por gestores, sejam eles de negócio ou TI. Os resultados indicaram uma maior predominância de gestores de negócios sobre os gestores de TI em ambas as cidades, porém em graus diferentes.

Quadro 5 - Tipo de gestor

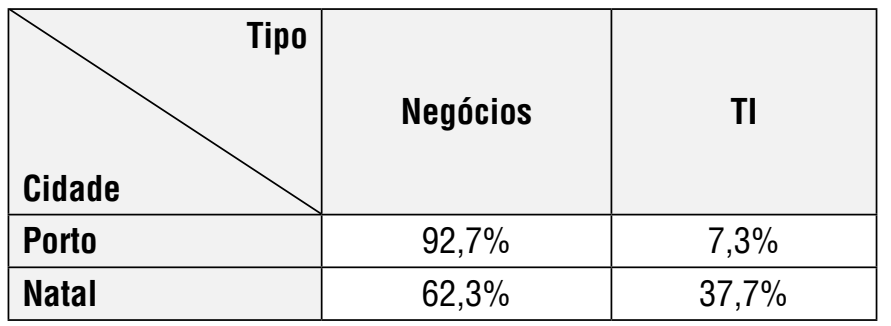

Fonte: Dados da pesquisa (2019)

0 perfil de empresas questionadas é, portanto, predominantemente formado por grandes empresas nas duas localidades em que foram aplicados, em sua maioria são negócios multinacionais em Porto e empresas nacionais em Natal. Quanto ao objeto de vendas das empresas há predominância de prestação de serviços em Porto, enquanto que em Natal obteve-se maioria em prestação de serviço juntamente com venda de produtos, o questionário foi respondido em maior porcentagem por gestores de negócios.

\subsection{Matriz importância (atual e futura) x desempenho}

\subsubsection{Capacidade dinâmica sensing}

De acordo com Teece (2007) o sensing é um microfundamento das capacidades dinâmicas, representa a capacidade de atuar analisando 0 ambiente externo na qual a organização está inserida, envolve processos para direcionar trabalhos internos de P\&D; processos de buscar fornecedores para complementar as inovações da organização; processos para buscar desenvolvimentos científicos e tecnológicos externos à firma e processos para reconhecer segmentos de mercado alvo, mudando as necessidades dos clientes e gerando inovações direcionadas para os clientes.

0 quadro 6 demonstra a média, mediana e moda obtidas por meio da escala do tipo Likert. A partir dos resultados, observa-se que dentre as tecnologias apresentadas, a que melhor foi avaliada 
quanto ao desempenho em ambas as cidades foi Big Data, seguido de IoT. 0 desempenho da inteligência artificial ainda é incipiente ou pouco explorado, principalmente na cidade de Natal. Por meio da observação dos dados, nota-se um maior grau de maturidade dessas tecnologias na Cidade do Porto comparado à cidade de Natal. Além disso, os dados apontam que essas tecnologias ainda são pouco utilizadas na análise do ambiente interno e externo à organização, uma vez que a escala variava de 1 a 7.

Quadro 6 - Comparação da capacidade dinâmica sensing com as tecnologias Big Data, IoT e IA.

\begin{tabular}{|c|c|c|c|c|c|c|c|}
\hline \multirow{2}{*}{ Tecnologia } & \multirow{2}{*}{ Dimensão } & \multicolumn{2}{|c|}{ Média } & \multicolumn{2}{|c|}{ Mediana } & \multicolumn{2}{|c|}{ Moda } \\
\hline & & Porto & Natal & Porto & Natal & Porto & Natal \\
\hline \multirow[b]{3}{*}{ IOT } & Importância & 5,13 & 3,79 & 5 & 4 & 5 & 1 \\
\hline & Desempenho & 3,50 & 2,11 & 4 & 2 & 4 & 1 \\
\hline & $\begin{array}{l}\text { Importância } \\
\text { (perspectiva } \\
\text { futura) }\end{array}$ & 5,93 & 5,01 & 6 & 5 & 7 & 1 \\
\hline \multirow[b]{3}{*}{ Big Data } & Importância & 5,47 & 4,42 & 6 & 5 & 6 & 3 \\
\hline & Desempenho & 3,97 & 3,58 & 4 & 4 & 4 & 4 \\
\hline & $\begin{array}{l}\text { Importância } \\
\text { (perspectiva } \\
\text { futura) }\end{array}$ & 6,03 & 5 & 6 & 5 & 7 & 6 \\
\hline \multirow[b]{3}{*}{ IA } & Importância & 4,55 & 3,04 & 5 & 3 & 5 & 2 \\
\hline & Desempenho & 2,15 & 1,83 & 2 & 1 & 2 & 1 \\
\hline & $\begin{array}{l}\text { Importância } \\
\text { (perspectiva } \\
\text { futura) }\end{array}$ & 5,36 & 4,87 & 6 & 5 & 7 & 7 \\
\hline
\end{tabular}

Fonte: Dado da pesquisa (2019)

A tecnologia Big Data é um meio de identificar e conhecer o perfil de consumidores de uma organização, para assim desenvolver um atendimento personalizado ao público (NOVO; AZEVED0, 2014). As informações obtidas por meio do Big Data possuem o poder de auxiliar nas tomadas de decisões, é utilizado também para o melhoramento de produtos ou serviços fornecidos e contribuir para a melhora da qualidade de vida (SILVA et al., 2013). Honorato, Carreira e Goveia (2014) acrescentam que a análise dessas informações pode possuir diversos objetivos, como compreender e identificar modelos comportamentais através de imagens obtidas em redes. Portanto, é irrefutável que o Big Data é uma ferramenta que possui ampla influência para obter vantagem competitiva (NOVO; NEVES, 2013; VOLPATO; RUFINO; DIAS, 2013; HENRIQUES; COSTA, 2014; MOURA; AMORIM, 2014;) 
Quanto à importância, os gestores reconhecem a importância dessas tecnologias, mesmo aquelas que apresentam baixo desempenho, como a IA. Conforme argumentam Kane et al. (2015), a capacidade de reinventar digitalmente o negócio é determinada em grande parte por uma estratégia digital clara, apoiada por líderes que promovem uma cultura capaz de mudar e inventar o novo. 0 que é relevante para a transformação digital é que a tomada de decisão sobre riscos, está se tornando uma cultura mais aceita à medida que mais empresas digitalmente avançadas buscam novos níveis de vantagem competitiva, através da inovação e introdução dessas tecnologias.

A principal diferença entre 0 comparativo das cidades está na importância futura esperada pelos gestores sobre as tecnologias. Na cidade de Natal, os gestores apontaram como perspectiva de importância futura maior no loT, seguido do Big Data. Esse fato não se repete na cidade do Porto, onde o Big Data representa maior expectativa dos gestores, seguido de loT. Apesar dessa diferença, em ambas as cidades, há crescimento em relação à importância dessas tecnologias na utilização em processos de análise do ambiente.

É perceptível que a capacidade dinâmica sensing relacionada com as tecnologias estudadas possui um baixo percentual de desempenho, em comparação à importância atual e perspectiva futura, em ambas as cidades, porém analisando o quadro de modo macro as empresas localizadas em Porto se destacam percentualmente, nas três dimensões de cada tecnologia pesquisada com exceção, em comparação às localizadas em Natal.

\subsubsection{Capacidade dinâmica seizing}

0 seizing é outro microfundamento que compõem as capacidades dinâmicas, ele está relacionado com a capacidade de aproveitar as chances e ou oportunidades detectadas, envolve processos voltados para soluções para o cliente; processos de seleção das fronteiras organizacionais; processos para seleção de protocolos de tomada de decisões; processos para construir lealdade de comprometimento e processos de descentralização e decomposição (TEECE, 2007).

No quadro 7, observa-se valores percentuais maiores nas três tecnologias, quando comparados as capacidades dinâmicas sensing. Essa informação pode indicar maior maturação e uso das três tecnologias na capacidade dinâmica seizing. Assim como na capacidade dinâmica anterior, o Big Data apresenta, na visão dos gestores, maior desempenho frente ao IoT e IA. 
Quadro 7 - Comparação da capacidade dinâmica seizing com as tecnologias Big Data, IoT e IA.

\begin{tabular}{|c|c|c|c|c|c|c|c|}
\hline \multirow{2}{*}{ Tecnologia } & \multirow{2}{*}{ Dimensão } & \multicolumn{2}{|c|}{ Média } & \multicolumn{2}{c|}{ Mediana } & \multicolumn{2}{c|}{ Moda } \\
\cline { 3 - 8 } & & Porto & Natal & Porto & Natal & Porto & Natal \\
\hline \multirow{4}{*}{ IOT } & Importância & 5,85 & 3,82 & 5 & 4 & 6 & 5 \\
\cline { 2 - 9 } & Desempenho & 4,10 & 3,53 & 4 & 4 & 4 & 4 \\
\cline { 2 - 8 } & $\begin{array}{c}\text { Importância } \\
\text { (perspectiva } \\
\text { futura) }\end{array}$ & 5,85 & 4,58 & 6 & 5 & 7 & 5 \\
\hline \multirow{5}{*}{ Big Data } & Importância & 5,32 & 4,47 & 6 & 5 & 6 & 5 \\
\cline { 2 - 9 } & Desempenho & 4,41 & 3,72 & 4 & 4 & 4 & 4 \\
\cline { 2 - 8 } & $\begin{array}{c}\text { Importância } \\
\text { (perspectiva } \\
\text { futura) }\end{array}$ & 5,93 & 5,21 & 6 & 6 & 7 & 7 \\
\hline \multirow{5}{*}{ IA } & Importância & 4,58 & 3,21 & 5 & 3 & 5 & 3 \\
\cline { 2 - 8 } & Desempenho & 3,18 & 1,72 & 3 & 1 & 3 & 1 \\
\cline { 2 - 8 } & $\begin{array}{c}\text { Importância } \\
\text { (perspectiva } \\
\text { futura) }\end{array}$ & 5,46 & 4,62 & 5 & 4 & 5 & 4 \\
\hline
\end{tabular}

Fonte: Dados da pesquisa (2019)

Há semelhança quanto à importância atual expressada pelos gestores sobres às tecnologias em ambas as cidades. Contudo, a respeito da expectativa de importância futura, na cidade do Porto, os gestores apontaram maior importância para as tecnologias Big Data com média de 5,93 e loT com média 5,85, enquanto que na cidade de Natal a expectativa de importância é apontada para Big Data, seguido de IA, com médias de 5,21 e 4,62, respectivamente.

A relação da capacidade dinâmica seizing com as tecnologias Big Data IoT e IA se demonstrou novamente, assim como no sensing, menor desempenho atual dentre as três dimensões. Além de empresas situadas em porto possuírem maior envolvimento tecnológico que as de Natal, com exceção da tecnologia Big Data a qual possui médias próximas nas três dimensões avaliadas em ambas as cidades.

\subsubsection{Capacidade dinâmica Managing Threats/Transforming}

Segundo Teece (2007) managing threats/transforming é a capacidade de gerenciar ameaças e transformações decorrentes das mudanças organizacionais, é a capacidade de se reconfigurar para se adaptar às demandas do mercado, envolve processos de coespecialização; processos de governança e processos de gerenciamento do conhecimento. 
No quadro 8, observa-se novamente a predominância de desempenho do BigData sobre as demais tecnologias, sendo seguida pelo IoT, em ambas as cidades. Nota-se mais uma vez o fraco desempenho apontado pelos gestores sobre a tecnologia IA, principalmente na cidade Natal, onde a mesma obteve média de 1,81.

Quadro 8 - Comparação da capacidade dinâmica managing threats/transforming com as tecnologias Big Data, IoT e IA.

\begin{tabular}{|c|c|c|c|c|c|c|c|}
\hline \multirow{2}{*}{ Tecnologia } & \multirow{2}{*}{ Dimensão } & \multicolumn{2}{|c|}{ Média } & \multicolumn{2}{c|}{ Mediana } & \multicolumn{2}{c|}{ Moda } \\
\cline { 3 - 8 } & & Porto & Natal & Porto & Natal & Porto & Natal \\
\hline \multirow{5}{*}{ IOT } & Importância & 5,26 & 3,60 & 5 & 4 & 5 & 1 \\
\cline { 2 - 8 } & Desempenho & 3,88 & 2,79 & 4 & 2 & 4 & 1 \\
\cline { 2 - 8 } & $\begin{array}{c}\text { Importância } \\
\text { (perspectiva } \\
\text { futura) }\end{array}$ & 5,55 & 4,55 & 6 & 5 & 6 & 6 \\
\hline \multirow{5}{*}{ Big Data } & Importância & 5,15 & 4,47 & 5 & 5 & 5 & 5 \\
\cline { 2 - 8 } & Desempenho & 4,09 & 3,54 & 4 & 3 & 4 & 1 \\
\cline { 2 - 8 } & $\begin{array}{c}\text { Importância } \\
\text { (perspectiva } \\
\text { futura) }\end{array}$ & 5,79 & 5,43 & 6 & 6 & 7 & 7 \\
\hline \multirow{5}{*}{ IA } & Importância & 4,60 & 3,45 & 5 & 3 & 5 & 2 \\
\cline { 2 - 8 } & Desempenho & 2,36 & 1,81 & 3 & 1 & 3 & 1 \\
\cline { 2 - 8 } & $\begin{array}{c}\text { Importância } \\
\text { (perspectiva } \\
\text { futura) }\end{array}$ & 5,34 & 4,72 & 6 & 5 & 7 & 7 \\
\hline
\end{tabular}

Fonte: Dados da pesquisa (2019)

A respeito da importância dessas tecnologias, observa-se a principal diferença entre as cidades. Na cidade do Porto, a tecnologia IoT obteve maior importância, seguido do BigData, com médias de 5,26 e 5,15, respectivamente. Porém, na cidade de Natal, nota-se uma inversão nessas definições, uma vez que a tecnologia Big Data obteve maior média de importância, seguido do loT, com médias de 4,47 e 3,60, respectivamente.

Sobre a importância futura que essas tecnologias representarão na visão dos gestores, observase uma semelhança entre as cidades sobre a tecnologia que obteve maior média, Big Data. Na tecnologia seguinte, há uma diferença: na cidade do Porto, a tecnologia IoT vem em seguida, porém, na cidade de Natal, a tecnologia que ocupa essa posição de importância é a IA.

0 quadro 9 demonstra a representação de onde estão as maiores médias de cada tecnologia por dimensão e um comparativo em ambas as cidades. Observa-se que na cidade do Porto, na dimensão 
importância atual, há uma predominância da capacidade dinâmica Seizing, em todas as tecnologias, demonstra que no cenário atual, as empresas localizadas em Porto, creditam importância da utilização das tecnologias para aproveitar as oportunidades de mercado, enquanto na cidade de Natal, esse mesmo fato não se repete.

Quadro 9: Comparativo de médias obtidas, tecnologia por dimensão.

\begin{tabular}{|c|c|c|c|c|c|}
\hline Cidade & Tecnologia & $\begin{array}{l}\text { Importância } \\
\text { atual }\end{array}$ & \multicolumn{2}{|c|}{ Desempenho } & $\begin{array}{c}\text { Importância } \\
\text { (perspectiva } \\
\text { futura) }\end{array}$ \\
\hline \multirow{3}{*}{ 옹 } & IOT & Seizing & \multicolumn{2}{|c|}{ Seizing } & Sensing \\
\hline & Big Data & Seizing & \multicolumn{2}{|c|}{ Sensing } & Sensing \\
\hline & IA & Seizing & \multicolumn{2}{|c|}{ Managing } & Seizing \\
\hline \multirow{3}{*}{$\frac{\bar{N}}{\frac{\pi}{2}}$} & IoT & Managing & \multicolumn{2}{|c|}{ Seizing } & Sensing \\
\hline & Big Data & Seizing & Seizing & Managing & Managing \\
\hline & IA & Sensing & \multicolumn{2}{|c|}{ Managing } & Sensing \\
\hline
\end{tabular}

Fonte: Dados da pesquisa (2019)

Além disso, outro ponto de destaque, em ambas as cidades, na dimensão importância futura, está na capacidade dinâmica Sensing, que predomina sobre as outras capacidades. Isso demonstra uma maior importância, atribuída pelos gestores, de que as tecnologias estudadas neste trabalho, apresentarão importância elevada na análise do ambiente e na busca por oportunidades. Através da análise da dimensão de desempenho infere-se que, empresas localizadas em Natal, utilizam as tecnologias para aproveitar as chances identificadas no ambiente e gerenciar as mudanças decorrentes do processo de reconfiguração.

\section{CONSIDERAÇÕES FINAIS}

A partir dos resultados, sobre a relação entre as tecnologias e as capacidades dinâmicas, sensing, seizing e managing, foi observado que a tecnologia Big Data foi a qual obteve maior percentual de desempenho atual sobre as demais tecnologias. Ademais, a tecnologia IA, foi a ferramenta tecnológica que obteve as menores médias nas três capacidades avaliadas, sendo pouco utilizado, principalmente em Natal. Analisando de forma macro, as empresas localizadas em Porto se sobressaem em comparação às localizadas em Natal, em todas as dimensões e capacidades. Contudo, quando avaliado a importância das tecnologias, atual e futura, foi detectado um potencial de desenvolvimento futuro no desempenho em todas as categorias analisadas, tanto em Porto como em Natal. 
Quando comparado médias obtidas, tecnologias por dimensão, foi detectado em Porto a capacidade dinâmica sensing se sobressai em todas as tecnologias, ao contrário de Natal, em que a importância atual é avaliada distribuída, cada capacidade correspondendo a uma tecnologia. Já na categoria desempenho, observa-se que em Natal há predominância das capacidades managing e seizing, enquanto que em Porto são distribuídas. Na perspectiva futura é destacada a capacidade sensing em ambas as localidades estudadas.

As empresas estão atravessando o processo de transformação digital e para Kane et al. (2015), as organizações estão criando habilidades para buscar a maturidade digital, e as que conseguem amadurecer digitalmente são quatro vezes mais propensas a fornecer aos colaboradores as habilidades necessárias para esse amadurecimento digital.

A pesquisa realizada foi relevante para comparar o desenvolvimento empresarial tecnológico em Portugal e no Brasil, demonstrando como está ocorrendo o processo de transformação digital nas diferentes localidades, e como essa transformação contribui para as capacidades dinâmicas. Assim sendo, o objetivo proposto inicialmente que era identificar as relações entre IoT, Big Data e a Inteligência Artificial com os microfundamentos das capacidades dinâmicas, em organizações localizadas na Cidade do Porto-PT e em Natal-BR, e comparar os resultados obtidos nas duas localidades foi alcançado.

\section{REFERÊNCIAS}

ALEEM, A.; RYAN SPROTT, C. Let me in the cloud: analysis of the benefit and risk assessment of cloud platform. Journal of Financial Crime, n. 20, v.1, p. 6-24, 2012.

\section{ALMEIDA, D. B.; MONÇÃO, L. D. S. Instituto Federal de Educação, Ciência e tecnologia} Fluminense, 2010.

ARNDT, F.; NORBERT, B. Evolutionary and Ecological conceptualization of dynamic capabilities: Identifying elements of the Teece and Eisenhardt schools. Journal of Management \& Organization, $p$. $1-4,2015$.

ASHTON, K. That "Internet of Things” Thing. RFiD Journal, 2009.

BADGER, L.; PATT-CORNER, R.; VOAS, J. Draft Cloud Computing Synopsis and Recommendations Recommendations of the National Institute of Standards and Technology. Nist Special Publication, v. 117, n. 84, 2011.

BALESTRO, M. V. et al. A experiência da rede PETRO-RS : uma estratégia para o desenvolvimento das capacidades dinâmicas. Revista de Administração Contemporânea (RAC), v. Ed. esp., 2004. 
BARRETO, I. Dynamic Capabilities: A Review of Past Research and an Agenda for the Future. Journal of Management, v. 36, n.1, p. 256-280, 2010.

BORBA, D. K.; SANTOS, L. F. A.; JUNIOR, L. T. K. Big Data : percepção dos usuários sobre vantagens e invasão de privacidade. VIII Workshop de Pós-Graduação e Pesquisa do Centro Paula Souza, p. 695-704, 2013.

BORGES, H. P. et al. Computação em nuvem. Instituto Brasileiro de Informação em Ciência e Tecnologia, 2011.

BRAGANZA, A. et al. Resource management in big data initiatives: Processes and dynamic capabilities. Journal of Business Research, v. 70, p. 328-337, 2017. https://doi.org/10.1016/j. jbusres.2016.08.006

BRETERNITZ, V. J.; SILVA, L. A. DA; LOPES, F. S. 0 uso de Big Data em Computacional social Science : tema que a sociedade precisa discutir. Faculdade de Computação e Informática Universidade Presbiteriana Mackenzie, 2013.

CALDAS, M. S.; SILVA, E. C. C. Fundamentos e aplicação do Big Data : como tratar informações em uma. Bibliotecas Universitárias: pesquisas, experiências e perspectivas, v. 3, p. 65-83, 2016.

CARAFFINI, J. P. T. S.; SOUZA, R. B. L.; BEHR, A. Transformação digital e desempenho no setor bancário. Congresso Transformação Digital 2018, p. 1-16, 2017.

COELHO, P. M. N. Rumo à Indústria 4.0. (Dissertação de mestrado). Coimbra p. 65, 2016.

CÔRTE-REAL, N.; OLIVEIRA, T.; RUIVO, P. Assessing business value of Big Data Analytics in European firms. Journal of Business Research, v. 70, p. 379-390, 2016.

DIAS, C. L. S. Carlos luís soares dias computação em nuvem. Instituto CEUB de Pesquisa e Desenvolvimento - ICPD, 2013.

DINH, T.; KIM, Y.; LEE, H. A Location-Based Interactive Model of Internet of Things and Cloud (IoTCloud) for Mobile Cloud Computing Applications. Sensors, v. 17, n. 3, p. 489, 2017.

DOBRE, C.; XHAFA, F. Intelligent services for Big data science. Future Generation Computer Systems, v, 37, p. 267-281, 2014.

EISENHARDT, K. M.; MARTIN, J. A. Dynamic capabilities: what are they? Strategic Management Journal, n. 21, p. 10-11, 2000.

EREVELLES, S.; FUKAWA, N.; SWAYNE, L. Big Data consumer analytics and the transformation of marketing. Journal of Business Research, v. 69, n 2, 2016.

GALDO, A. M. R. Capacidades Dinâmicas para a Inovação Aberta: análise com base no Capital intelectual. Repositório Institucional da Universidade Federal de Santa Catarina, n. 8791, 2016.

GALEGALE, G. P. et al. Internet das Coisas aplicada a negócios: Um estudo bibliométrico. Journal of Information Systems and Technology Management, v. 13, n. 3, 2016. 
GUBBI, J. et al. Internet of Things (IoT): A vision, architectural elements, and future directions. Future Generation Computer Systems, v. 29, n. 7, 2013.

GUERRA, R. M. A.; TONDOLO, V. A. G.; CAMARGO, M. E. 0 que (ainda) podemos aprender sobre capacidades dinâmicas. Revista Ibero Americana de Estratégia, v. 15, p. 44-64, 2016.

HART, J. K.; MARTINEZ, K. Toward an environmental Internet of Things. Earth and Space Science, v. 2, n. 5, 2015.

HELFAT, C. E.; PETERAF, M. A. Understanding dynamic capabilities: progress along a developmental path. So! Apbox Editorial Essays, v. 7, n. 1, p. 91-102, 2009.

HENRIQUES, M. S. B. et al. 123 4. Revista Pensar Tecnologia, v. 2, 2013.

HENRIQUES, D. A.; COSTA, H. R. Big Data - Como utilizar a extraordinária quantidade de informações coletadas por novas tecnologias para obter vantagens competitivas. Revista Pensar Tecnologia, v. 3 , p. 1-11, 2014.

HERMANN, M.; PENTEK, T.; OTTO, B. Design Principles for Industrie 4.0 Scenarios: A Literature Review, 2015.

HERRMANN, J. C. D. A influencia das capacidades dinâmicas no processo de contínui ajustamento estratégico. Faculdade de Administração, Contabilidade e Economia, PUCRS, p. 55-60, 2013.

HESS, T.; et al. Options for Formulating a Digital Transformation Strategy. MIS Quarterly Executive, v. 15, n. 2, p. 123-139, 2016.

HONORATO, J. I.; CARREIRA, L. S.; GOVEIA, F. G. Intercom - Sociedade Brasileira de Estudos Interdisciplinares da Comunicação. XIX Congresso de Ciências da Comunicação na Região Sudeste, v. 2, p. 1-14, 2014.

JUNIOR, J. C. S. F. et al. Dimensões de Big Data e o Processo Decisório: Estudos de Casos Múltiplos no Varejo, 2015.

KANE, G. C. et al. Strategy, Not Technology, Drives Digital Transformation. MIT Sloan Management Review, 2015.

KLASHANOV, F. Artificial Intelligence and Organizing Decision in Construction. Procedia Engineering, 2016.

LUI, F.; et al. NIST Cloud Computing Reference Architecture: Recommendations of the National Institute of Standards and Technology. NIST Special Publication 500-292, 2011.

MAÇADA, A. C. G.; CANARY, V. P. A Tomada de Decisão no Contexto do Big Data: estudo de caso único. XXXVIII Encontro da ANPAD - EnANPAD, XXXVIII, 2014.

MACADAR, M. A.; GARCIA, P. S. Abordagens Teóricas Sobre a Temática do IoT na Gestão da Informação. Curitiba: ENADI 2017. 
MACEDO, J. F. S. G. A. Computação em nuvem - Um estudo empírico exploratório sobre as determinantes da preocupação com a proteção de dados. Instituto Superior de Contabilidade e Administração do Porto, 2015.

MAKRIDAKIS, S. The Forthcoming Artificial Intelligence (AI) Revolution: Its Impact on Society and Firms, 2017.

MALHOTRA, N. K. Pesquisa de Marketing: uma orientação aplicada (3 ed.). Porto Alegre: Bookman, 2001.

MARSTON, S. et al. Cloud computing - The business perspective. Decision Support Systems, 2011.

MARTILLA, J. A.; JAMES, J. C. (1977). Importance-Performance Analysis. Journal of Marketing, 1997.

MCAFEE, A.; BRYNJOLFSSON, E. Big Data : The Management Revolution. Har, (OctOBeR), 2012.

MEIRELLES, D. S. E.;CAMARGO, Á. A. B. Capacidades Dinâmicas : 0 Que São e Como Identificálas ? Dynamic Capabilities: What Are They and How to Identify Them ? Revista de Administração Contemporânea, 2014.

MELL, P.; GRANCE, T. The NIST Definition of Cloud Computing Recommendations of the National Institute of Standards and Technology, 2010.

MOURA, A. L. T.; AMORIM, D. G. Big Data: 0 impacto e sua Funcionalidade na Sociedade. Revista Opara: Ciências Contemporâneas Aplicadas, v. 4, 2014.

NOVO, R.; NEVES, J. M. S. Inovação na inteligência analítica por meio do Big Data : características de diferenciação da abordagem tradicional. VIII Workshop de Pós-graduação e Pesquisa do Centro Paula Souza, p. 32-44, 2013.

NOVO, R.; AZEVEDO, M. M. Análise de dados. Big Data. Ética. Informação. Redes Sociais. Tekhne e Logos, p. 64-78, 2014.

OLIVEIRA, A. L. et al. Computação em Nuvens. Revista de Trabalhos Acadêmicos, v. 7, 2013.

PEDROSA, P. H. C.; NOGUEIRA, T. Computação em Nuvem. Universidade Estadual de Campinas, p. 3-6, 2011.

PEREIRA, ALANA D. S. A formação das capacidades dinâmicas por meio da gestão de indicativos de conhecimento. Biblioteca Karl A. Boedecker da Fundação Getúlio Vargas -SP, p. 1-203, 2018.

PIMENTA, R. M. Big data e controle da informação na era digital: tecnogênese de uma memória a serviço do mercado e do Estado. Tendências da Pesquisa Brasileira em Ciência da Informação, v. 6, 2013.

ROTTA, C. Capacidades dinâmicas e desempenho inovador: uma análise dos setores químico e eletroeletrônico brasileiros. Repositório Digital da Biblioteca da Universidade do Vale do Rio dos Sinos, 2011. 
RUSCHEL, H.; ZANOTTO, M. S.; MOTA, W. C. DA. Computação em Nuvem Henrique. Pontifícia Universidade Católica do Paraná, 2010.

SAKTHIVEL, R.; PARTHIPAN, V.; DHANASEKARAN, D. Big Data Analytics on Smart and Connected Communities Using Internet of Things. International Journal of Pharmacy \& Technology, 2016.

SANTOS, U.; AMELOTTI, L. A.; VILLAR, F. Adoção de Computação em Nuvem e suas Motivações. Cloud Security Alliance (CSA), 2012.

SCHROEDER, R. Big data: moldando o conhecimento, moldando a vida cotidiana 1. University of Oxford, Oxford Internet Institute, p. 135-163, 2018.

SCHWAB, K. A Quarta Revolução Industrial (Edipro). São Paulo, 2016.

SILVA, T. L. C. et al. Minicurso 1. Simpósio Brasileiro de Banco de Dados, p. 1-26, 2013.

SIVARAJAH, U. et al. Critical analysis of Big Data challenges and analytical methods. Journal of Business Research, 2017.

SOUSA, F. R. C.; MOREIRA, L. 0.; MACHADO, J. C. Computação em Nuvem: Conceitos, Tecnologias, Aplicações e Desafios. Escola Regional de Computação Ceará Maranhão e Piauí - ERCEMAPI, 2009.

TEECE, D. J. Explicating Dynamic Capabilities: The Nature and Microfoundations of (Sustainable) Enterprise Performance. Strategic Management Journal, 2007.

TEECE, D. J.; PISANO, G.; SHUEN, A. M. Y. Dynamic Capabilities and Strategic Management Author ( S ): David J . Teece , Gary Pisano and Amy Shuen Published by : Wiley Stable, 1997. Disponivel: http://www.jstor.org/stable/3088148 MANAGEMENT.

TESSAROLO, P. H.; MAGALHÃES, W. B. A era do Big Data no conteúdo digital: os dados estruturados e não estruturado. Universidade Paranaense (UNIPAR), 2014.

TONDOLO, V. A. G.; BITENCOURT, C. Compreendendo as Capacidades Dinâmicas a Partir de Seus Antecedentes, Processos e Resultados. Brazilian Business Review, v. 11, p. 124-147, 2014.

VAN KRANENBURG, R.; ANZELMO, E. The Internet of Things. 1st Berlin Symposium on Internet and Society, (October), 2008.

VenKATRAM, K.; GEETHA, M. A. Review on Big Data \&amp; Analytics - Concepts, Philosophy, Process and Applications. Cybernetics and Information Technologies, 2017.

VERAS, M. Cloud Computing: Nova Arquitetura da TI. Rio de Janeiro: Brasport, 2012.

VIEIRA, C. S.; MEIRELLES, F. D. S. use. Revista Eletrônica Gestão e Serviços, v. 6, p. 1215-1230, 2015.

VOLPATO, T.; RUFINO, R. R.; DIAS, J. W. Big Data - Transformando dados em decisões.

Universidade Paranaense (UNIPAR), 2013. 
WAMBA, S. F. et al. Big data analytics and firm performance: Effects of dynamic capabilities. Journal of Business Research, 70, 356-365, 2017.

WOLFERT, S. et al. Big Data in Smart Farming ? A review. Agricultural Systems, 2017.

YANAI, A. E. et al. 0 desenvolvimento da indústria 4.0: um estudo bibliométrico. XXXVII Encontro Nacional de Engenharia de Produção, 2018.

ZHU, C. et al. Green Internet of Things for Smart World. IEEE Access, 2015. 